PROCEEDINGS OF THE

AMERICAN MATHEMATICAL SOCIETY

Volume 140, Number 10, October 2012, Pages 3313-3322

S 0002-9939(2012)11174-8

Article electronically published on January 31,2012

\title{
CENTRAL VALUES \\ OF THE SYMMETRIC SQUARE $L$-FUNCTIONS
}

\author{
WENZHI LUO
}

(Communicated by Matthew A. Papanikolas)

Abstract. We establish a sharp bound for the square mean of the central values of the symmetric square $L$-functions associated to holomorphic cusp forms of level 1 , as the weight $k$ varies in the short interval $\left[K, K+K^{1 / 2+\epsilon}\right]$.

\section{INTRODUCTION}

Let $S_{k}\left(\Gamma_{0}(1)\right)$ denote as usual the space of holomorphic cusp forms of weight $k$ with respect to the modular group $\Gamma_{0}(1)$, and let $H_{k}$ be the normalized Hecke basis for $S_{k}\left(\Gamma_{0}(1)\right)$, where the normalizaton means that the first Fourier coefficient of each basis element $f \in H_{k}$ is 1 .

Each $f \in H_{k}$ has the Fourier expansion

$$
f(z)=\sum_{n \geq 1} a_{f}(n) n^{(k-1) / 2} e(n z), \quad a_{f}=1,
$$

and the associated $L$-function

$$
L(s, f)=\sum_{n \geq 1} a_{f}(n) n^{-s}=\prod_{p}\left(1-\alpha_{p} p^{-s}\right)^{-1}\left(1-\beta_{p} p^{-s}\right)^{-1}, \Re(s)>1,
$$

admits analytic continuation to the whole complex plane $\mathbf{C}$ and satisfies the functional equation

$$
(2 \pi)^{-s} \Gamma\left(\frac{k-1}{2}+s\right) L(s, f)=i^{k}(2 \pi)^{1-s} \Gamma\left(\frac{k-1}{2}+1-s\right) L(1-s, f) .
$$

The symmetric square $L$-function associated to $f$ is defined by

$$
L\left(s, \operatorname{sym}^{2}(f)\right)=\prod_{p}\left(1-\alpha_{p}^{2} p^{-s}\right)^{-1}\left(1-\alpha_{p} \beta_{p} p^{-s}\right)^{-1}\left(1-\beta_{p}^{2} p^{-s}\right)^{-1}, \Re(s)>1,
$$

and by the works of Shimura [7] and Gelbart-Jacquet [1, it admits analytic continuation to the whole complex plane $\mathbf{C}$ and satisfies the functional equation

$$
\begin{aligned}
\Lambda\left(s, \operatorname{sym}^{2}(f)\right) & =: \pi^{-3 s / 2} \Gamma\left(\frac{s+1}{2}\right) \Gamma\left(\frac{s+k-1}{2}\right) \Gamma\left(\frac{s+k}{2}\right) L\left(s, \operatorname{sym}^{2}(f)\right) \\
& =\Lambda\left(1-s, \operatorname{sym}^{2}(f)\right) .
\end{aligned}
$$

Write

$$
L_{\infty}(s)=L_{\infty}\left(s, \operatorname{sym}^{2}(f)\right)=\pi^{-3 s / 2} \Gamma\left(\frac{s+1}{2}\right) \Gamma\left(\frac{s+k-1}{2}\right) \Gamma\left(\frac{s+k}{2}\right)
$$

Received by the editors February 10, 2011 and, in revised form, March 31, 2011. 2010 Mathematics Subject Classification. Primary 11F11, 11F66, 11F67.

The author's research was partially supported by NSF grant DMS-0855600. 
and note that

$$
L\left(s, \operatorname{sym}^{2}(f)\right)=\zeta(2 s) \sum_{n \geq 1} a_{f}\left(n^{2}\right) n^{-s}, \Re(s)>1 .
$$

To study the central $L$-value $L\left(1 / 2, \operatorname{sym}^{2}(f)\right)$, the following approximate functional equation is a standard device (see, for example, [5, Lemma 2.2] for a detailed proof).

Lemma 1 ([5]). We have

$$
L\left(1 / 2, \operatorname{sym}^{2}(f)\right)=2 \sum_{n \geq 1} \frac{a_{f}\left(n^{2}\right)}{n^{1 / 2}} V_{k}(n),
$$

where

$$
V_{k}(\xi)=\frac{1}{2 \pi i} \int_{(\sigma)} \frac{L_{\infty}(1 / 2+y)}{L_{\infty}(1 / 2)} \zeta(1+2 y) \xi^{-y} \frac{d y}{y}(\sigma>0),
$$

and it satisfies the following bounds:

$$
V_{k}(\xi) \ll_{A}\left(\frac{k}{\xi}\right)^{A}, \text { for any } A>0,
$$

and

$$
V_{k}(\xi) \ll \log (2 k / \xi), \text { for } \xi \leq k .
$$

The purpose of this paper is to show

Theorem 1. For any $\epsilon>0$ we have, as $K \rightarrow \infty$,

$$
\sum_{2 \mid k, K \leq k \leq K+K^{1 / 2}} \sum_{f \in H_{k}}\left|L\left(1 / 2, \operatorname{sym}^{2}(f)\right)\right|^{2} \ll_{\epsilon} K^{3 / 2+\epsilon} .
$$

Remark. It seems to be an open problem (see, for instance, Conjecture 1.2 on p. 5 of [5]) to obtain the sharp bound for the square mean without average over the weight $k$. Previously the work [3] established a sharp bound for the second moment for $L\left(1 / 2, \operatorname{sym}^{2}(f)\right)$, when $f$ has fixed weight and large level, but its method unfortunately does not extend to the case of large weight. Our work is inspired by [6], which makes use of the Zagier kernel function $\Phi_{s}$ for $L\left(s, \operatorname{sym}^{2}(f)\right)$ to derive a sharp bound for the first moment.

\section{ZAGIER'S KERNEL FUNCTION}

Let $\Delta$ be a discriminant; i.e., $\Delta$ is an integer such that $\Delta \equiv 0,1(\bmod 4)$. Define

$$
L(s, \Delta)= \begin{cases}\zeta(2 s-1), & \text { if } \Delta=0 ; \\ L_{D}(s) \sum_{d \mid f} \mu(d)\left(\frac{D}{d}\right) d^{-s} \sigma_{1-2 s}\left(\frac{f}{d}\right), & \text { if } \Delta \neq 0,\end{cases}
$$

where if $\Delta \neq 0$ we write $\Delta=D f^{2}$ with positive integer $f$ and $D$ the fundamental discriminant, $L_{D}(s)$ is the Dirichlet $L$-function associated to the Kronecker symbol $\left(\frac{D}{.}\right), \mu$ is the Möbius function, and $\sigma_{\nu}(m)=\sum_{d \mid m} d^{\nu}$ for a positive integer $m$ and any complex number $\nu$. 
Moreover for $t$ an integer with $\Delta<t^{2}$ and $s \in \mathbf{C}$ with $1 / 2<\Re(s)<k$ we define (see [8])

$$
\begin{aligned}
I_{k}(\Delta, t ; s) & =\int_{0}^{\infty} \int_{-\infty}^{\infty} \frac{y^{k+s-2}}{\left(x^{2}+y^{2}+i t y-\frac{1}{4} \Delta\right)^{k}} d x d y \\
& =\frac{\Gamma(k-1 / 2) \Gamma(1 / 2)}{\Gamma(k)} \int_{0}^{\infty} \frac{y^{k+s-2}}{\left(y^{2}+i t y-\frac{1}{4} \Delta\right)^{k-1 / 2}} d y,
\end{aligned}
$$

where the second integral converges absolutely for $1-k<\sigma<k$ if $\Delta \neq 0$. For $\Delta=0, \pm t>0$, one has

$$
I_{k}(0, t ; s)=e^{ \pm i \pi(s-k) / 2} \frac{\Gamma(1 / 2) \Gamma(s-1 / 2) \Gamma(k-s)}{\Gamma(k)}|t|^{-k+s} .
$$

We also have

$$
2 I_{k}(\Delta, 0,1 / 2)=\frac{1}{m^{k-1 / 2}} \frac{\Gamma\left(\frac{k-1 / 2}{2}\right)^{2} \Gamma(1 / 2)}{\Gamma(k)} .
$$

Define

$$
I_{k, s}(z)=\frac{2^{1-k} \Gamma(1 / 2)}{\Gamma(k-1 / 2)} \Gamma(k-1+s) \Gamma(k-s)\left(z^{2}-1\right)^{-(k-1) / 2} P_{-s}^{1-k}(z),
$$

for $1-k<\Re(s)<k, z \in \mathbf{C} \backslash(-\infty, 1]$, where $P_{\nu}^{\mu}(z)$ is the associated Legendre function of the first kind. Then for $\Delta<0$, it follows that

$$
I_{k}(\Delta, t ; s)=(|\Delta| / 4)^{(s-k) / 2} \frac{\Gamma(k-1 / 2) \Gamma(1 / 2)}{\Gamma(k)} I_{k, s}\left(\frac{i t}{\sqrt{|\Delta|}}\right),
$$

while for $\Delta>0$, the following formulas are valid:

$$
\begin{aligned}
& I_{k}(\Delta, t ; s)=(\Delta / 4)^{(s-k) / 2} e^{i \pi(s-k) / 2} \frac{\Gamma(k-1 / 2) \Gamma(1 / 2)}{\Gamma(k)} I_{k, s}\left(\frac{t}{\sqrt{\Delta}}\right) \quad(\Delta>0, t>0) ; \\
& I_{k}(\Delta, t ; s)=(\Delta / 4)^{(s-k) / 2} e^{-i \pi(s-k) / 2} \frac{\Gamma(k-1 / 2) \Gamma(1 / 2)}{\Gamma(k)} I_{k, s}\left(\frac{|t|}{\sqrt{\Delta}}\right) \quad(\Delta>0, t<0) .
\end{aligned}
$$

We refer the reader to $\left[\underline{8}\right.$ for the proofs of these formulas for $I_{k}(\Delta, t ; s)$. Now we are in a position to state the following important theorem due to Zagier, which is the basis for our study on the square mean of $L\left(1 / 2, \operatorname{sym}^{2}(f)\right)$ via the kernel function $\Phi_{s}$.

Theorem 2 ([8]). Let $k \geq 4$ be an even integer. For a positive integer $m$ and $s \in \mathbf{C}$ set

$$
\begin{aligned}
c_{m}(s)= & m^{k-1} \sum_{t=-\infty}^{\infty}\left(I_{k}\left(t^{2}-4 m, t ; s\right)+I_{k}\left(t^{2}-4 m,-t ; s\right)\right) L\left(s, t^{2}-4 m\right) \\
& + \begin{cases}(-1)^{k / 2} \frac{\Gamma(k+s-1) \zeta(2 s)}{2^{2 s+k-3} \pi^{s-1} \Gamma(k)} u^{k-s-1}, & \text { if } m=u^{2}, u>0 ; \\
0, & \text { if } m \text { is not a perfect square. }\end{cases}
\end{aligned}
$$

Then:

(i) The series converges absolutely and uniformly for $2-k<\Re(s)<k-1$.

(ii) The function

$$
\Phi_{s}(z)=\sum_{m=1}^{\infty} c_{m}(s) e(m z) \quad(z \in \mathbf{H}, 2-k<\Re(s)<k-1)
$$

is in $S_{k}\left(\Gamma_{0}(1)\right)$. 
(iii) Let $f \in H_{k}$. Then the Petersson product of $\Phi_{s}$ and $f$ is given by

$$
\left\langle\Phi_{s}, f\right\rangle=c_{k} \frac{\Gamma(s+k-1)}{(4 \pi)^{s+k-1}} L\left(s, \operatorname{sym}^{2}(f)\right),
$$

where

$$
c_{k}=\frac{(-1)^{k / 2} \pi}{2^{k-3}(k-1)} .
$$

In the above theorem, if $m$ is a square, then the single term outside the sum has a simple pole at $s=1 / 2$, while in this case the terms $t= \pm 2 \sqrt{m}$ in the first sum involve the function

$$
I_{k}(0, t, s)+I_{k}(0, t, s)=2 \pi(-1)^{k / 2} \cos \frac{\pi s}{2} \frac{\Gamma(s-1 / 2) \Gamma(k-s)}{\Gamma(k) \Gamma(1 / 2)}|t|^{s-k},
$$

which also has a simple pole at $s=1 / 2$, and the two poles cancel, so that $c_{m}(s)$ is actually holomorphic in the region $2-k<\Re(s)<k-1$.

We infer that

$$
\Phi_{s}(z)=c_{k} \frac{\Gamma(s+k-1)}{(4 \pi)^{s+k-1}} \sum_{f \in H_{k}} \frac{L\left(s, \operatorname{sym}^{2}(f)\right)}{\langle f, f\rangle} f(z) .
$$

Taking the $m$-th Fourier coefficient of both sides, we have

$$
c_{m}(s)=c_{k} \frac{\Gamma(s+k-1)}{(4 \pi)^{s+k-1}} \sum_{f \in H_{k}} \frac{L\left(s, \operatorname{sym}^{2}(f)\right)}{\langle f, f\rangle} m^{(k-1) / 2} a_{f}(m) .
$$

Specializing at $s=1 / 2$ and replacing $m$ by $m^{2}$, it follows from Lemma 1 that

$$
2 \sum_{m \geq 1} \frac{c_{m^{2}}(1 / 2)}{m^{k-1 / 2}} V_{k}(m)=c_{k} \frac{\Gamma(k-1 / 2)}{(4 \pi)^{k-1 / 2}} \sum_{f \in H_{k}} \frac{\left|L\left(1 / 2, \operatorname{sym}^{2}(f)\right)\right|^{2}}{\langle f, f\rangle} .
$$

Recall that

$$
\langle f, f\rangle=\frac{\Gamma(k) L\left(1, \operatorname{sym}^{2}(f)\right)}{2^{2 k-1} \pi^{k+1}} .
$$

Hence

(2)

$\sum_{f \in H_{k}} \frac{\left|L\left(1 / 2, \operatorname{sym}^{2}(f)\right)\right|^{2}}{L\left(1, \operatorname{sym}^{2}(f)\right)}=(-1)^{k / 2} 2^{-2} \pi^{-5 / 2}\left(\frac{\Gamma(k-1 / 2)}{2^{k}(k-1) \Gamma(k)}\right)^{-1} \sum_{m \geq 1} \frac{c_{m^{2}}(1 / 2)}{m^{k-1 / 2}} V_{k}(m)$.

\section{The CASE $\Delta>0$}

We need the following integral representation of $P_{\nu}^{-\mu}(z)$ ([2, p. 1001, 8.713 (2)]):

$$
P_{\nu}^{-\mu}(z)=\frac{\left(z^{2}-1\right)^{\mu / 2}}{2^{\nu} \Gamma(\mu-\nu) \Gamma(\nu+1)} \int_{0}^{\infty} \frac{\sinh ^{2 \nu+1} r}{(z+\cosh r)^{\nu+\mu+1}} d r
$$

for $\Re(z)>-1,|\arg (z \pm 1)|<\pi, \Re(\nu+1)>0, \Re(\mu-\nu)>0$.

Consider first the case where $\Delta=t^{2}-4 m^{2}>0$. With $z=\frac{|t|}{\sqrt{t^{2}-4 m^{2}}}$ we deduce that

$$
\left|P_{-1 / 2}^{1-k}(z)\right| \ll \frac{\left|z^{2}-1\right|^{(k-1) / 2}}{\Gamma(k-1 / 2)} \int_{0}^{\infty} \frac{1}{(z+\cosh r)^{k-1 / 2}} d r
$$


Now for any $\delta>0$,

$$
\begin{aligned}
& \int_{0}^{\infty} \frac{1}{(z+\cosh r)^{k-1 / 2}} d r \\
& \ll \frac{1}{(z+1)^{k-1 / 2-\delta}} \int_{0}^{\infty} \frac{1}{(\cosh r)^{\delta}} d r \\
& \ll_{\delta} \frac{1}{(z+1)^{k-1 / 2-\delta}} \\
& \ll_{\delta} \frac{\left(t^{2}-4 m^{2}\right)^{(k-1 / 2-\delta) / 2}}{\left(|t|+\sqrt{t^{2}-4 m^{2}}\right)^{(k-1 / 2-\delta)}} \\
& \ll \delta\left\{\begin{array}{lr}
\frac{1}{(1+1 / \sqrt{2})^{k}} \frac{\left(t^{2}-4 m^{2}\right)^{(k-1 / 2-\delta) / 2}}{\left.|t|\right|^{(k-1 / 2-\delta)}} & \text { if }|t|>\sqrt{8} m, \\
\frac{\left(t^{2}-4 m^{2}\right)^{(k-1 / 2-\delta) / 2}}{|t|^{(k-1 / 2-\delta)}(1+\sqrt{1-2 m /|t|})^{(k-1 / 2-\delta)}} & \text { if } 2 m<|t| \leq \sqrt{8} m
\end{array}\right. \\
& \ll_{\delta} \frac{1}{\exp \left(k^{1 / 3}\right)} \frac{\left(t^{2}-4 m^{2}\right)^{(k-1 / 2) / 2}}{|t|^{(k-1 / 2-\delta)}},
\end{aligned}
$$

since in view of $|t| \geq 2 m+1$, we have

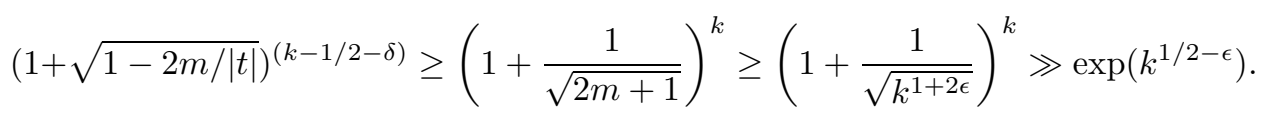

Note for $\Delta=D f^{2}$ as before, the convexity bound yields

$$
|L(1 / 2, \Delta)| \ll f^{\epsilon}\left|L_{D}(1 / 2)\right| \ll \Delta^{1 / 4+\epsilon} .
$$

Furthermore,

$$
\begin{aligned}
& \left|I_{k, 1 / 2}(z)\right| \ll 2^{-k} \Gamma(k-1 / 2)\left|z^{2}-1\right|^{-(k-1) / 2}\left|P_{-1 / 2}^{1-k}(z)\right| \\
& \ll 2^{-k} \int_{0}^{\infty} \frac{1}{(z+\cosh r)^{k-1 / 2}} d r, \\
& \left|I_{k}(\Delta, t, 1 / 2)\right| \ll(\Delta / 4)^{(1 / 2-k) / 2} \frac{\Gamma(k-1 / 2)}{\Gamma(k)}\left|I_{k, 1 / 2}(|t| / \sqrt{\Delta})\right| \\
& \ll \Delta^{-(k-1 / 2) / 2} \frac{\Gamma(k-1 / 2)}{\Gamma(k)} \int_{0}^{\infty} \frac{1}{(|t| / \sqrt{\Delta}+\cosh r)^{k-1 / 2}} d r \\
& \ll \frac{\Gamma(k-1 / 2)}{\exp \left(k^{1 / 3}\right)|t|^{(k-1 / 2-\delta)} \Gamma(k)} .
\end{aligned}
$$

Hence the contribution to the right-hand side of (2) from the terms with $\Delta=$ $t^{2}-4 m^{2}>0$ is

$$
\begin{aligned}
\ll & k^{-10}+\left(\frac{\Gamma(k-1 / 2)}{2^{k} k \Gamma(k)}\right)^{-1} \sum_{m \ll k^{1+\epsilon}} \frac{m^{2 k-2}}{m^{k-1 / 2}} \sum_{|t|>2 m} \\
& \times\left(I_{k}\left(t^{2}-4 m^{2}, t ; 1 / 2\right)+I_{k}\left(t^{2}-4 m^{2},-t ; 1 / 2\right)\right) L\left(1 / 2, t^{2}-4 m^{2}\right)
\end{aligned}
$$

$(3) \ll 1$ 


\section{THE CASE $\Delta \leq 0$}

Next we consider the terms in the right-hand side of (2) with $\Delta=t^{2}-4 m^{2}<0$ and $z=\frac{i t}{\sqrt{|\Delta|}}$. We have

$$
\begin{aligned}
I_{k}(\Delta, t ; s)= & (|\Delta| / 4)^{(1 / 2-k) / 2} \frac{\Gamma(k-1 / 2) \Gamma(1 / 2)}{\Gamma(k)} I_{k, 1 / 2}\left(\frac{i t}{\sqrt{|\Delta|}}\right) \\
= & (|\Delta| / 4)^{(1 / 2-k) / 2} \frac{\Gamma(k-1 / 2) \Gamma(1 / 2)}{\Gamma(k)} \\
& \times 2^{1-k} \Gamma(1 / 2) \Gamma(k-1 / 2)\left(z^{2}-1\right)^{-(k-1) / 2} P_{-1 / 2}^{1-k}(z) \\
= & 2^{1-k}(|\Delta| / 4)^{(1 / 2-k) / 2} \frac{\Gamma^{2}(k-1 / 2) \Gamma^{2}(1 / 2)}{\Gamma(k)}\left(z^{2}-1\right)^{-(k-1) / 2} \\
& \times \frac{\left(z^{2}-1\right)^{(k-1) / 2}}{\left.2^{-1 / 2} \Gamma(k-1 / 2)\right) \Gamma(1 / 2)} \int_{0}^{\infty} \frac{1}{(z+\cosh r)^{k-1 / 2}} d r \\
= & 2^{3 / 2-k} \Gamma(1 / 2)(|\Delta| / 4)^{(1 / 2-k) / 2} \frac{\Gamma(k-1 / 2)}{\Gamma(k)} \int_{0}^{\infty} \frac{1}{(z+\cosh r)^{k-1 / 2}} d r \\
= & 2 \Gamma(1 / 2)|\Delta|^{(1 / 2-k) / 2} \frac{\Gamma(k-1 / 2)}{\Gamma(k)} \int_{0}^{\infty} \frac{1}{(z+\cosh r)^{k-1 / 2}} d r .
\end{aligned}
$$

Making the change of variable $u=\cosh t$, we have

$$
\begin{aligned}
\int_{0}^{\infty} \frac{1}{(z+\cosh r)^{k-1 / 2}} d r & =\int_{1}^{\infty} \frac{d u}{\sqrt{u^{2}-1}(z+u)^{k-1 / 2}} \\
& =\int_{0}^{\infty} \frac{d u}{\sqrt{u^{2}+2 u}(z+1+u)^{k-1 / 2}}
\end{aligned}
$$

Applying Cauchy's theorem we can replace the integral over the positive real line by the line $\{(1+z) v, v \geq 0\}$, and the above integral equals (we may assume $m \ll k^{1+\epsilon}$ )

$$
\begin{aligned}
& \frac{1}{(1+z)^{k-1}} \int_{0}^{\infty} \frac{d v}{\sqrt{v}(1+v)^{k-1 / 2} \sqrt{2+(1+z) v}} \\
& =\frac{1}{(1+z)^{k-1}}\left(\int_{0}^{k^{\epsilon-1}} \frac{d v}{\sqrt{v}(1+v)^{k-1 / 2} \sqrt{2+(1+z) v}}+O\left(k^{-A}\right)\right) \\
& =\frac{1}{\sqrt{2}(1+z)^{k-1}}\left(\int_{0}^{k^{\epsilon-1}} \frac{d v}{\sqrt{v}(1+v)^{k-1 / 2}}+O\left(k^{-1+\epsilon}\right)\right) \\
& =\frac{\sqrt{2}|\Delta|^{(k-1) / 2}}{2^{k} m^{k-1}}\left(\frac{1+z}{|1+z|}\right)^{-(k-1)}\left(\int_{0}^{k^{\epsilon-1}} \frac{d v}{\sqrt{v}(1+v)^{k-1 / 2}}+O\left(k^{-1+\epsilon}\right)\right),
\end{aligned}
$$


in view of

$$
|1+z|=\left(\frac{2 m}{\sqrt{|\Delta|}}\right)
$$

and

$$
|\Delta|=4 m^{2}-t^{2}=(2 m-t)(2 m+t)>m .
$$

Hence

$$
\begin{aligned}
I_{k}(\Delta, t ; s)= & 2 \Gamma(1 / 2)|\Delta|^{(1 / 2-k) / 2} \frac{\Gamma(k-1 / 2)}{\Gamma(k)} \int_{0}^{\infty} \frac{1}{(z+\cosh r)^{k-1 / 2}} d r \\
= & 2 \sqrt{2} \Gamma(1 / 2)|\Delta|^{-1 / 4} \frac{\Gamma(k-1 / 2)}{2^{k} \Gamma(k)} \frac{1}{m^{k-1}}\left(\frac{1+z}{|1+z|}\right)^{-(k-1)} \\
& \times\left(\int_{0}^{k^{\epsilon-1}} \frac{d v}{\sqrt{v}(1+v)^{k-1 / 2}}+O\left(k^{-1+\epsilon}\right)\right) .
\end{aligned}
$$

Thus the contribution to the right-hand side of (2) from the terms with $\Delta=$ $t^{2}-4 m^{2}<0$ is

$(4)$

$$
\begin{aligned}
& O\left(k^{-10}\right)+(-1)^{k / 2} 2^{-2} \pi^{-5 / 2}\left(\frac{\Gamma(k-1 / 2)}{2^{k}(k-1) \Gamma(k)}\right)^{-1} \sum_{m \ll k^{1+\epsilon}} \frac{m^{2 k-2}}{m^{k-1 / 2}} V_{k}(m) \\
& \times \sum_{|t|<2 m}\left(I_{k}\left(t^{2}-4 m^{2}, t ; 1 / 2\right)+I_{k}\left(t^{2}-4 m^{2},-t ; 1 / 2\right)\right) L\left(1 / 2, t^{2}-4 m^{2}\right) \\
& =k^{-10}+(-1)^{k / 2} 2^{-1 / 2} \pi^{-2}(k-1) \sum_{m \ll k^{1+\epsilon}} m^{-1 / 2} V_{k}(m) \sum_{|t|<2 m}\left(4 m^{2}-t^{2}\right)^{-1 / 4} \\
& \times\left(\left(\frac{\sqrt{4 m^{2}-t^{2}}+t i}{\left|\sqrt{4 m^{2}-t^{2}}+t i\right|}\right)^{-(k-1)}+\left(\frac{\sqrt{4 m^{2}-t^{2}}-t i}{\left|\sqrt{4 m^{2}-t^{2}}-t i\right|}\right)^{-(k-1)}\right) L\left(1 / 2, t^{2}-4 m^{2}\right) \\
& \times\left(\int_{0}^{k^{\epsilon-1}} \frac{d v}{\sqrt{v}(1+v)^{k-1 / 2}}+O\left(k^{-1+\epsilon}\right)\right) \\
& =O\left(k^{1+\epsilon}\right)+(-1)^{k / 2} 2^{-1 / 2} \pi^{-2}(k-1)\left(\int_{0}^{k^{\epsilon-1}} \frac{d v}{\sqrt{v}(1+v)^{k-1 / 2}}\right) \\
& \times \sum_{m \ll k^{1+\epsilon}} m^{-1 / 2} V_{k}(m) \sum_{|t|<2 m}\left(4 m^{2}-t^{2}\right)^{-1 / 4} \\
& \times\left(\left(\frac{\sqrt{4 m^{2}-t^{2}}+t i}{\left|\sqrt{4 m^{2}-t^{2}}+t i\right|}\right)^{-(k-1)}+\left(\frac{\sqrt{4 m^{2}-t^{2}}-t i}{\left|\sqrt{4 m^{2}-t^{2}}-t i\right|}\right)^{-(k-1)}\right) L\left(1 / 2, t^{2}-4 m^{2}\right) .
\end{aligned}
$$


In the last step, we have inferred as follows:

$$
\begin{aligned}
& \sum_{m \ll k^{1+\epsilon}} m^{-1 / 2} \sum_{|t|<2 m}\left(4 m^{2}-t^{2}\right)^{-1 / 4} L\left(1 / 2, t^{2}-4 m^{2}\right) \\
& \ll k^{\epsilon} \sum_{2^{i} \leq k^{1+\epsilon}} \frac{1}{2^{i / 2}} \sum_{f \leq 2^{i+1}} \frac{1}{f^{1 / 2}} \sum_{|D| \leq 2^{2 i+2} / f^{2}} \frac{\left|L_{D}(1 / 2)\right|}{|D|^{1 / 4}} \\
& \ll k^{\epsilon} \sum_{2^{i} \leq k^{1+\epsilon}} \frac{1}{2^{i / 2}} \sum_{f \leq 2^{i+1}} \frac{1}{f^{1 / 2}}\left(\sum_{|D| \leq 2^{2 i+2} / f^{2}}\left|L_{D}(1 / 2)\right|^{2}\right)^{1 / 2}\left(\sum_{|D| \leq 2^{2 i+2} / f^{2}}|D|^{1 / 2}\right)^{1 / 2} \\
& \ll k^{\epsilon} \sum_{2^{i} \leq k^{1+\epsilon}} \frac{2^{3 i / 2}}{2^{i / 2}} \sum_{f \leq 2^{i+1}} \frac{1}{f^{2}} \\
& \ll k^{1+3 \epsilon},
\end{aligned}
$$

where we have used Jutila's bound 4,

$$
\sum_{|D| \leq X}\left|L_{D}(1 / 2)\right|^{2} \ll X \log ^{3} X .
$$

Moreover the contribution to the right-hand side of (2) from the terms with $\Delta=t^{2}-4 m^{2}=0$, i.e. $t= \pm 2 m$, is

$$
\begin{aligned}
& \ll k^{-10}+\left(\frac{\Gamma(k-1 / 2)}{2^{k} k \Gamma(k)}\right)^{-1} \sum_{m \ll k^{1+\epsilon}} \frac{m^{2 k-2}}{m^{k-1 / 2}} \frac{\Gamma(k-1 / 2)}{2^{k} m^{k-1 / 2} \Gamma(k)} \log ^{2} k \\
& \ll k \log ^{3} k,
\end{aligned}
$$

in view of the bound

$$
\frac{\Gamma^{\prime}(s)}{\Gamma(s)}=\log s+O(1 / s), \text { for } \Re(s) \geq 1 .
$$

\section{The Mean Value in the Short interval}

The contribution from (4) is bounded trivially by $k^{3 / 2+\epsilon}$, but if we average over $k$ with $K \leq k \leq K+K^{1 / 2}$, then the total contribution will be shown below to be $O\left(K^{3 / 2+\epsilon}\right)$.

When $K \leq k \leq K+K^{1 / 2}$, the equality (4) can be replaced by (in light of the formula (2.7) in [5] for $\left.V_{k}(\xi)\right)$

$$
\begin{aligned}
& =O\left(K^{1+\epsilon}\right)+(-1)^{k / 2} 2^{-1 / 2} \pi^{-2} K\left(\int_{0}^{K^{\epsilon-1}} \frac{d v}{\sqrt{v}(1+v)^{K-1 / 2}}\right) \\
& \times \sum_{m \ll K^{1+\epsilon}} m^{-1 / 2} V_{K}(m) \sum_{|t|<2 m}\left(4 m^{2}-t^{2}\right)^{-1 / 4} \\
& \times\left(\left(\frac{\sqrt{4 m^{2}-t^{2}}+t i}{\left|\sqrt{4 m^{2}-t^{2}}+t i\right|}\right)^{-(k-1)}+\left(\frac{\sqrt{4 m^{2}-t^{2}}-t i}{\left|\sqrt{4 m^{2}-t^{2}}-t i\right|}\right)^{-(k-1)}\right) L\left(1 / 2, t^{2}-4 m^{2}\right) .
\end{aligned}
$$


Now we are summing over all even $k$ in $(6)$ with $K \leq k \leq K+K^{1 / 2}$. Denote

$$
e^{i \theta_{t, m}}=\frac{\sqrt{4 m^{2}-t^{2}}+t i}{\left|\sqrt{4 m^{2}-t^{2}}+t i\right|}
$$

and since

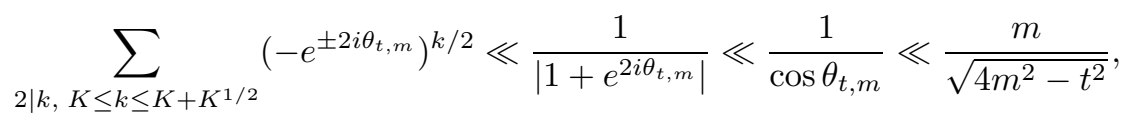

we have, with $t^{2}-4 m^{2}=D f^{2}$,

$$
\begin{aligned}
& \sum_{m \ll K^{1+\epsilon}} m^{-1 / 2} V_{K}(m) \sum_{|t|<2 m}\left(4 m^{2}-t^{2}\right)^{-1 / 4} \\
& \times\left(\left(\frac{\sqrt{4 m^{2}-t^{2}}+t i}{\left|\sqrt{4 m^{2}-t^{2}}+t i\right|}\right)^{-(k-1)}+\left(\frac{\sqrt{4 m^{2}-t^{2}}-t i}{\left|\sqrt{4 m^{2}-t^{2}}-t i\right|}\right)^{-(k-1)}\right) L\left(\frac{1}{2}, t^{2}-4 m^{2}\right) \\
\ll & K^{\epsilon} \sum_{m \ll K^{1+\epsilon}} m^{1 / 2} \sum_{|t|<2 m}\left(4 m^{2}-t^{2}\right)^{-3 / 4}\left|L\left(1 / 2, t^{2}-4 m^{2}\right)\right| \\
\ll & K^{1 / 2+\epsilon} \sum_{m \ll K^{1+\epsilon}} \sum_{|t|<2 m}\left(4 m^{2}-t^{2}\right)^{-3 / 4}\left|L_{D}(1 / 2)\right| \\
\ll & K^{1 / 2+\epsilon} \sum_{f \ll K^{1+\epsilon}} f^{-3 / 2} \sum_{|D| \ll K^{2+\epsilon}}|D|^{-3 / 4}\left|L_{D}(1 / 2)\right| \ll K^{1+\epsilon} .
\end{aligned}
$$

Hence we conclude that

$$
\sum_{2 \mid k, K \leq k \leq K+K^{1 / 2}} \sum_{f \in H_{k}}\left|L\left(1 / 2, \operatorname{sym}^{2}(f)\right)\right|^{2} \ll_{\epsilon} K^{3 / 2+\epsilon} .
$$

This completes the proof of Theorem 1.

\section{ACKNOWLEDGEMEnts}

Part of this work was carried out during the author's visit to the Beijing International Center for Mathematical Research at the Peking University during the summers of 2009 and 2010. He wishes to thank the Institute for the generous support and for the excellent environment to work in. The author would like to thank the referee for a thorough reading of this paper and for some very valuable comments and suggestions.

\section{REFERENCES}

[1] S. Gelbart, H. Jacquet, A relation between automorphic representations of $G L(2)$ and $G L(3)$, Ann. Sci. Ecole Norm. Sup. $4^{e}$, série 11, 1978, 471-552. MR533066 (81e:10025)

[2] I. S. Gradshtein, I. M. Ryzhik, Tables of Integrals, Series and Products, Academic Press, New York and London, 1965. MR0197789 (33:5952)

[3] H. Iwaniec, P. Michel, The second moment of the symmetric square L-functions, Ann. Acad. Sci. Fenn. Math. 26, 2001, 465-482. MR 1833252 (2002d:11051)

[4] M. Jutila, On the mean value of $L(1, \chi)$ for real characters, Analysis 1, 1981, 149-161. MR632705 (82m:10065)

[5] R. Khan, Non-vanishing of the symmetric square L-function at the central point, Proc. Lond. Math. Soc. (3) 100, 2010, 736-762. MR2640289 (2011e:11085) 
[6] W. Kohnen, J. Sengupta, On the average of central values of symmetric square L-functions in weight aspect, Nagoya Math. J. 167, 2002, 95-100. MR1924720 (2003g:11050)

[7] G. Shimura, On the holomorphy of certain Dirichlet series, Proc. London Math. Soc. (3) 31, 1975, 79-98. MR0382176 (52:3064)

[8] D. Zagier, Modular forms whose Fourier coefficients involve zeta-functions of quadratic fields, Modular Functions of One Variable. VI, Lecture Notes in Math. 627, 105-169, Springer, Berlin-Heidelberg-New York, 1977. MR0485703 (58:5525)

Department of Mathematics, The Ohio State University, 231 West 18th Avenue, Columbus, Оhiо 43210

E-mail address: wluo@math.ohio-state.edu 